\title{
Preimplantation Genetic Diagnosis for Congenital Immunodeficiencies
}

\section{Anver Kuliev* and Svetlana Rechitsky}

Reproductive Genetics Institute, Chicago, IL, USA

\begin{abstract}
Preimplantation genetic diagnosis [PGD] is presently a realistic option for at risk couples to avoid affected pregnancies and have a genetic disease free offspring of their own. PGD has been also performed together with HLA typing for couples with children affected by congenital disorders, requiring the HLA identical stem cell transplantation therapy. As the HLA matched stem cell transplantation is known to significantly improve or completely replenish the immune system, PGD was applied as an alternative treatment strategy for congenital immunodeficiencies, so that the couples could have unaffected children, who are also HLA compatible to serve as stem cell donor progeny for the affected siblings. We present here our experience of PGD cycles for inherited immunodeficiencies, which is presently the world's largest series of PGD for this group of diseases, for which there is still no available treatment other than stem cell transplantation.
\end{abstract}

Keywords: Preimplantation genetic diagnosis; Preimplantation HLA typing; congenital immunodeficiencies; HLA identical stem cell transplantation

\section{Introduction}

Preimplantation genetic diagnosis [PGD] has been performed for approximately four hundred different conditions, resulting in birth of thousands healthy children free of genetic disorders [1-3]. PGD has been also applied for preimplantation HLA typing as a treatment tool for the affected siblings with genetic and acquired disorders, requiring HLA matched stem cell transplantation [4-9]. The first application of this approach was reported for Fanconi anemia [4], which was then applied also for siblings with other genetic and acquired disorders [5-9].

It is well established that the HLA matched stem cell transplantation improves or completely replenishes the immune system, so PGD is a potential alternative for preventing inherited forms of immunodeficiency, to ensure the birth of unaffected children, as a potential stem cell donor progeny for their affected siblings. Feasibility of successful use of PGD for immunodeficiencies has previously been demonstrated for Wiscott-Aldrich Syndrome [WAS], X-linked hyper IgM syndrome [HIGM], and X-linked hypohidrotic ectodermal dysplasia with immune deficiency [HED-ID] [4-9]. The practical usefulness of the approach for preventing the birth of children with immunodeficiencies, as well as transplantation treatment of sibling, requiring stem cell transplantation as the only available treatment, is demonstrated below, which is currently the world's largest experience in PGD for immunideficiencies.

\section{Material and Methods}

\section{PGD cycles}

A total of 82 PGD cycles were performed for 36 couples at risk for producing immunodeficiencient progeny, including 20 cycles for HIGM, 20 for incontinentia pigmenti [IP], 20 for hyper-IgM type 1 immunodeficiency [HIGM1], 10 for chronic X-linked granulomatous disease [CGD], 9 for hypohidrotic ectodermal dysplasia with immune deficiency [HED-ID], 7 for Wiscott-Aldrich syndrome [WAS], 6 for Omenn syndrome [OMS], 5 for Type 1 X-linked agammaglobulenemia, 3 for ataxia-telangiectasia [AT], and 2 for $\mathrm{X}$-linked immunodysregulation, polyendocrinopathy and enteropathy [IPEX] (Table 1).

\section{Biopsy procedures}

PGD cycles were performed using a standard IVF protocol coupled with micromanipulation procedures for the polar body [PB], or embryo biopsy, described in detail elsewhere [10]. The first PB [PB1] and second $\mathrm{PB}[\mathrm{PB} 2]$ were removed sequentially following maturation and fertilization of the oocytes using mechanical method, which is safe and highly efficient although laser assisted techniques may also be applied. After PB1 removal, the oocytes were inseminated with motile sperm, or using introcytoplasmic sperm injection [ICSI], and examined for the presence of pronuclei and extrusion of $\mathrm{PB} 2$, which were removed in the same manner as PB1. The biopsied oocytes were then returned to culture, checked for cleavage and transferred, depending on the genotype of the corresponding $\mathrm{PB} 1$ and $\mathrm{PB} 2$. It is understood that the $\mathrm{PB}$ approach allows testing only for maternally derived mutations, making possible to preselect unaffected embryos without a further testing for paternal mutations. However, HLA typing cannot be done on PB and requires embryo biopsy to obtain HLA typing information from both parents.

Embryo biopsy was performed initially in cleaving embryos, but currently mainly on the blastocyst stage both for identification of immunodeficiency mutations of paternal origin, heterozygous embryos originating from oocytes with maternal mutations, and for HLA testing, in order to identify the embryos containing the maternal and paternal chromosomes 6 identical to the sibling with immunodeficiencies. Blastomere biopsy was performed by a mechanical method, while blastocyst sampling was done by laser [10].

\section{Genetic analysis}

To avoid misdiagnosis due to preferential amplification, multiplex

*Corresponding author: Anver Kuliev, Reproductive Genetics Institute, 2910 MacArthur Boulevard, Northbrook, Chicago, IL 60062, USA, Tel: 847-400-1515 Fax: 847-400-1516; E-mail: anverkuliev@hotmail.com

Received July 25, 2014; Accepted October 25, 2014; Published November 15 2014

Citation: Kuliev A, Rechitsky S (2014) Preimplantation Genetic Diagnosis for Congenital Immunodeficiencies. J Blood Disorders Transf 5: 234. doi: 10.4172/21559864.1000234

Copyright: (C) 2014 Kuliev A, et al. This is an open-access article distributed under the terms of the Creative Commons Attribution License, which permits unrestricted use, distribution, and reproduction in any medium, provided the original author and source are credited. 


\begin{tabular}{|c|c|c|c|c|c|c|c|}
\hline Disease & Gene & \#Patient & \#Cycle & \#Transfers & \#Embryo transferred & Pregnancy \% & Birth \\
\hline Ataxia-telangiectasia; at & ATM & 1 & 3 & 2 & 3 & 1 & 1 \\
\hline Agammaglobulinemia, X-linked, Type I & BTK & 3 & 5 & 5 & 11 & 2 & 3 \\
\hline HLA + Immunodeficiency with Hyper-IgM, Type 1; HIGM1 & CD40LG & 6 & 11 & 7 & 11 & 4 & 3 \\
\hline Immunodeficiency with hyper-IgM, Type 1; HIGM1 & CD40LG & 4 & 9 & 9 & 13 & 3 & 3 \\
\hline HLA + granulomatous disease, chronic, $X$-linked; CGD & CYBB & 4 & 10 & 7 & 10 & 3 & 2 \\
\hline $\begin{array}{l}\text { Immunodysregulation, polyendocrinopathy, and } \\
\text { enteropathy, X-linked; IPEX }\end{array}$ & FOXP3 & 1 & 2 & 2 & 2 & 0 & 0 \\
\hline $\begin{array}{c}\text { HLA + ectodermal dysplasia, hypohidrotic, with immune } \\
\text { deficiency }\end{array}$ & IKBKG & 2 & 9 & 6 & 8 & 2 & 3 \\
\hline Incontinentia pigmenti; IP & IKBKG & 9 & 20 & 13 & 24 & 2 & 3 \\
\hline Omenn Syndrome (OMS) & RAG1 & 2 & 6 & 5 & 12 & 1 & 2 \\
\hline Wiskott-aldrich syndrome; was & WAS & 4 & 7 & 7 & 14 & 5 & 5 \\
\hline Total & & 36 & 82 & $63(71.4 \%)^{*}$ & $108(1.71)^{* *}$ & $23(36.5 \%)$ & 25 \\
\hline
\end{tabular}

* \% of cycles with embryo transfer

${ }^{\star *}$ Average number of embryos transferred

Table 1: Preimplantation Genetic Diagnosis for Congenital Immunodeficiences.

nested PCR was performed, involving a simultaneous detection of the mutant gene together with up to three or more highly polymorphic markers, closely linked to the gene tested. Only when the polymorphic sites and the mutation agreed, were embryos transferred. So multiplex amplification allowed detecting allele-drop out [ADO] and prevented the transfer of misdiagnosed affected embryos [10].

HLA genes were tested simultaneously, using the short tandem repeats in the HLA region, by applying a multiplex hemi-nested PCR system, involving only closely linked polymorphic short tandem repeat [STR] markers located throughout HLA region [D6S426, D6S291, Ring 3 CA, TAP1, G51152, D6S2447, LH1,DN, D6S273, 9N-2, TNF a,b,c,d; 62, MIC A, MIB, D6S276, D6S439, D6S1624, D6S265, D6S510; D6S248, RF, MOG a,b,c,d, D6S 258, D6S306, D6S464, D6S299, D6S461]. The choice of alleles and markers was based on the information they provide about the presence of maternal and paternal matching or non-matching chromosomes. For each family heterozygous alleles and markers were selected not shared by the parents. Such markers provided the information about the origin of chromosome 6. A haplotype analysis for father, mother and the affected child was performed for each family prior to preimplantation HLA typing, to avoid misdiagnosis due to preferential amplification and allele drop out [ADO], potential recombination within the HLA region. The probability of identifying unaffected embryos fully matched to thalassemic siblings is $18.75 \%$, based on the $25 \%$ and the $75 \%$ probabilities of HLA match and having unaffected embryos, respectively.

The HLA matched and immunodeficiency free embryos, based on the information about the mutation testing and polymorphic markers, were pre-selected for transfer back to patient, while non matched unaffected embryos were frozen for future use by the couple.

\section{Results and Discussion}

The results of PGD for immunodeficiencies are presented in Table 1. A total of 108 unaffected and HLA matched embryos were detected for transfer in 63 [71\%] of 82 PGD cycles for 36 couples carrying the above mentioned immunodeficiency mutations, resulting in 23 unaffected HLA matched [36.5\% per embryo transfer] pregnancies, which yielded the birth of 25 healthy children. The low average number of embryos transferred in these cycles [1.71 embryos per transfer] is due to a simultaneous pre-selection of HLA type, performed in 20 of 36 cycles, with the majority of them with advanced reproductive age. Overall, 29 embryos were unaffected and HLA matched to the siblings with immunodeficiencies and transferred in 20 PGD cycles, resulting in birth of 8 healthy HLA identical children, whose stem cells were transferred to the affected immunodeficient siblings, resulting in a complete cure.

The presented data show the usefulness of PGD for congenital immunodeficiencies, as there is no effective treatment for these conditions other than stem cell transplantation. PGD provides the couples at risk with the option to avoid the affected pregnancy and have a progeny free of disease. If they have already an affected immunodeficient child, PGD with HLA typing makes it also possible to have an access to the HLA identical stem cell transplantation through selection and transfer of those unaffected embryos which are also HLA match to the sibling. Because the finding of the HLA identical stem cell donor is the key for achieving the success in stem cell transplantation [11], a complete cure was observed in our cases after stem cell transplantation in siblings, which is in agreement with the results of another large series reported from Turkey [12-14].

Although ethical issues [15] still present a barrier for a wider application of this technology, there has been an increase in referral for PGD with preimplantation HLA typing, with the present overall world's experience of preimplantation HLA typing of more than thousand cases, the majority of which have been done within the last few years. The need for preimplantation HLA typing may be expected to increase in the future with the progress in cellular therapy, requiring the improvement of the access to HLA compatible stem cell transplantation.

\section{References}

1. Preimplantation Genetic Diagnosis International Society (PGDIS) (2008) Guidelines for good practice in PGD: program requirements and laboratory quality assurance. Reprod BioMed Online 16: 134-147.

2. ESHRE Preimplantation Genetic Diagnosis (PGD) Consortium (2011) Best practice guidelines for preimplantation genetic diagnosis/screening (PGD/ PGS). Hum Reprod 26: 14-46.

3. Kuliev A (2013) Practical preimplantation genetic diagnosis. Second Edition Springer, New York, London, Heidelberg, USA

4. Verlinsky Y, Rechitsky S, Schoolcraft W, Strom C, Kuliev A (2001) Preimplantation diagnosis for Fanconi anemia combined with HLA matching JAMA 285: 3130-3133

5. Verlinsky Y, Rechitsky S, Sharapova T, Morris R, Taranissi M, et al. (2004) Preimplantation HLA testing. JAMA 291: 2079-2085.

6. Rechitsky S, Kuliev A, Tur-Kaspa I, Morris R, Verlinsky Y (2004) Preimplantation HLA typing with Preimplantation genetic diagnosis. Reproductive BioMedicine Online 6: 488-493. 
Citation: Kuliev A, Rechitsky S (2014) Preimplantation Genetic Diagnosis for Congenital Immunodeficiencies. J Blood Disorders Transf 5: 234. doi: $10.4172 / 2155-9864.1000234$

Page 3 of 4

7. Kuliev A, Rechitsky S, Verlinsky O, Tur-Kaspa I, Kalakoutis G, et al. (2005) Preimplantation diagnosis and HLA typing for haemoglobin disorders. Reprod Biomed Online 11: 362-370.

8. Rechitsky S, Kuliev A, Sharapova T, Laziuk K, Ozen S, et al. (2006) Preimplantation HLA typing with aneuploidy testing. Reprod Biomed Online 12: 89-100.

9. Verlinsky Y, Rechitsky S, Sharapova T, Laziuk K, Barsky I, et al. (2007) Preimplantation diagnosis for immunodeficiencies. Reprod Biomed Online 14: 214-223.

10. Kuliev A, Rechitsky S, Verlinsky O (2014) Atlas of Preimplantation Genetic Diagnosis, (3rd Edn.), CRS Press, Taylor and Franis, London.

11. Gaziev J, Lucarelli G (2010) Allogeneic cellular gene therapy for hemoglobinopathies. Hematol Oncol Clin North Am 24: 1145-1163.
12. Kahraman S, Karlikaya G, Sertyel S, Karadayi H, Findikli N (2004) Clinical aspects of preimplantation genetic diagnosis for single gene disorders combined with HLA typing. Reprod Biomed Online 9: 529-532.

13. Kahraman S, Beyazyurek C, Ekmekci CG (2011) Seven years of experience of preimplantation HLA typing: a clinical overview of 327 cycles. Reprod Biomed Online 23: 363-371.

14. Kahraman S (2013) PGD for HLA: Clinical Outcomes of HLA compatible transplantation following PGD. Reprod Biomed Online 26 (Suppl 1): S9-S10.

15. Edwards RG (2004) Ethics of PGD: thoughts on the consequences of typing HLA in embryos. Reprod Biomed Online 9: 222-224. 